J. HONG

KODAI MATH. ].

17 (1994), $341-350$

\title{
TIMELIKE SURFACES WITH MEAN CURVATURE ONE IN ANTI-DE SITTER 3-SPACE
}

\author{
BY JIANQIAO HONG
}

\section{Introduction}

It is well-known that the classical Weierstrass-Enneper representation formula describes minimal surfaces in Euclidean 3-space $R^{3}$ in terms of their Gauss maps and auxiliary holomorphic functions [7]. This representation formula plays a very important role in constructing and studying minimal surfaces in $R^{3}$. Later, D. A. Hoffman and R. Osserman obtained the higher dimensional version of the classical Weierstrass-Enneper representation formula for minimal surfaces in Euclidean $n$-space $R^{n}$ [3]. A natural question is how to generalize the above results to the surfaces in space forms of other constant curvature. In 1987, R. L. Bryant gave a representation formula for surfaces of mean curvature one in hyperbolic 3-space $H^{3}[1]$. In his paper, he also pointed out that the surfaces of constant mean curvature in $S^{3}$ have no representation in terms of holomorphic data.

While considering the surfaces in Lorentz space forms, O. Kobayashi represented spacelike maximal surfaces in Lorentz-Minkowski 3-space $R_{1}^{3}$ in terms of holomorphic data [5]. Also C. H. Gu obtained the representation formula for the timelike and mixed type extremal surfaces in $R_{1}^{3}$ [2].

Motivated by these results, in this paper we obtain a reprsentation formula for timelike surfaces with mean curvature one in 3-dimensional anti-de Sitter $H_{1}^{3}$. By this formula, we get some timelike surfaces with mean curvature one in $H_{1}^{3}$.

This paper is organized as follows. In section 2 , we introduce the standard model of $H_{1}^{3}$, and set up another model of $H_{1}^{3}$ which is quite useful for computation. In section 3, we will prove the main theorems (Theorem 3.1 and Theorem 3.3) which describe the timelike surfaces of mean curvature one in $H_{1}^{3}$ in terms of two simple mappings. At last, in section 4, after writing the representation formula into a suitable form, we will give some examples.

\section{Models for $\boldsymbol{H}_{1}^{3}$}

On the 4-dimensional real vecter space $E^{4}$, we consider the symmetric form

Received June 30, 1993; revised November 24, 1993. 


$$
\begin{gathered}
\langle x, y\rangle=x_{1} y_{1}+x_{2} y_{2}-x_{3} y_{3}-x_{4} y_{4}, \\
x=\left(x_{1}, \cdots, x_{4}\right) \in E^{4}, \quad y=\left(y_{1}, \cdots, y_{4}\right) \in E^{4} .
\end{gathered}
$$

The pair $\left(E^{4},\langle\cdot, \cdot\rangle\right)$ will be denoted by $R_{2}^{4}$. Define $H_{1}^{3}$ as follows:

$$
H_{1}^{3}=\left\{x \in R_{2}^{4} ;\langle x, x\rangle=-1\right\} .
$$

When we consider $H_{1}^{3}$ with the induced pseudo-metric from $R_{2}^{4}$, it is easily shown that $H_{1}^{3}$ is a complete 3-dimensional, pseudo-Riemannian manifold of constant sectional curvature -1 and has signature $(+,+,-)$. One may refer to [8] for more detail to understand the completeness and other properties of anti-de Sitter 3-space.

Besides the above standard model for $H_{1}^{3}$, there is another way of describing $H_{1}^{3}$ which will be quite useful in our calculations. We identify $R_{2}^{4}$ with the space of $2 \times 2$ real matrices by identifying $\left(x_{1}, x_{2}, x_{3}, x_{4}\right)$ with the matrix

$$
\left(\begin{array}{cc}
x_{1}+x_{4} & x_{2}-x_{3} \\
x_{2}+x_{3} & -x_{1}+x_{4}
\end{array}\right)
$$

The real Lie group, $S L(2, R) \times S L(2, R)$, two copies of $2 \times 2$ real matrices with determinant 1 , acts naturally on $R_{2}^{4}$ by the representation

$$
\left(g_{1}, g_{2}\right) \cdot v=g_{1} v g_{2}^{t}
$$

where we regard $v$ as a $2 \times 2$ real matrix by (2.2). Under this identification, we clearly have $\langle v, v\rangle=-\operatorname{det} v$. Thus $S L(2, R) \times S L(2, R)$ preserves $\langle\cdot, \cdot\rangle$ and $H_{1}^{3}$ can be recognized as the space $S L(2, R)$

$$
H_{1}^{3}=\{g \in \operatorname{gl}(2, R): \operatorname{det} g=1\} .
$$

Let $\mathscr{F}$ be the oriented orthonormal frame bundle of $R_{2}^{4}$ which consists of the bases $\left(e_{1}, e_{2}, e_{3}, e_{4}\right)$ of $R_{2}^{4}$ satisfying conditions:

$$
\begin{gathered}
e_{1} \wedge e_{2} \wedge e_{3} \wedge e_{\boldsymbol{4}}>0, \\
\left\langle e_{\alpha}, e_{\beta}\right\rangle=\varepsilon_{\alpha} \delta_{\alpha \beta}
\end{gathered}
$$

where $\varepsilon_{1}=\varepsilon_{2}=1$ and $\varepsilon_{3}=\varepsilon_{4}=-1$. We can use $S L(2, R) \times S L(2, R)$ to parametrize $\mathcal{F}$ as follows.

Assume that

$$
\underline{e}_{1}=\left(\begin{array}{cc}
1 & 0 \\
0 & -1
\end{array}\right), \quad \underline{e}_{2}=\left(\begin{array}{ll}
0 & 1 \\
1 & 0
\end{array}\right), \quad \underline{e}_{3}=\left(\begin{array}{cc}
0 & -1 \\
1 & 0
\end{array}\right), \quad \underline{e}_{4}=\left(\begin{array}{ll}
1 & 0 \\
0 & 1
\end{array}\right),
$$

and let $e_{\alpha}\left(g_{1}, g_{2}\right)=\left(g_{1}, g_{2}\right) \cdot \underline{e}_{\alpha}=g_{1} \underline{e}_{\alpha} g_{2}^{t}$. Then the map $\left(g_{1}, g_{2}\right) \mapsto\left(e_{1}\left(g_{1}, g_{2}\right), \cdots\right.$, $\left.e_{4}\left(g_{1}, g_{2}\right)\right)$ is a $2-1$ covering map of $S L(2, R) \times S L(2, R)$ onto $\mathscr{F}$.

By submersion $e_{4}: \mathscr{F} \rightarrow H_{1}^{3}$, we may regard $\mathscr{I}$ as the oriented orthonormal frame bundle of $H_{1}^{3}$ such that $e_{1}, e_{2}, e_{3} \in T_{e_{4}} H_{1}^{3}$ is an orthonormal frame of $T_{e_{4}} H_{1}^{3}$. Denoting its dual frame fields by $\left\{\omega^{1}, \omega^{2}, \omega^{3}\right\}$. Then there exist unique 1-forms 
on $H_{1}^{3},\left\{\omega_{j}^{2} \mid \imath, j=1,2,3\right\}$ so that

$$
\begin{aligned}
& d e_{4}=\sum \omega^{2} e_{\imath}, \\
& d e_{\imath}=\sum \omega_{i}^{\jmath} e_{j}+\omega^{2} e_{4}, \\
& \omega_{i}^{\jmath} \varepsilon_{j}+\omega_{j}^{2} \varepsilon_{\imath}=0 .
\end{aligned}
$$

Denoting the metric on $H_{1}^{3}$ by $d s^{2}$, we have

$$
d s^{2}=\left\langle d e_{4}, d e_{4}\right\rangle=\left(\omega^{1}\right)^{2}+\left(\omega^{2}\right)^{2}-\left(\omega^{3}\right)^{2} .
$$

For an orthonormal frame on $H_{1}^{3}$ given by $\left\{e_{i}\left(g_{1}, g_{2}\right) \mid i=1,2,3\right\}$. The canonical forms $\left\{\omega^{2}, \omega_{j}^{\imath} \mid \imath, \jmath=1,2,3\right\}$ can be expressed by Maurer-Cartan forms of $g_{1}$ and $g_{2}$.

LEMMA 2.1. Let $\left.\left\{\omega^{\imath}, \omega_{j}^{\imath} \mid \imath,\right\}=1,2,3\right\}$ be the canonical forms associated with the frame $\left\{e_{\imath}=g_{1} \underline{e}_{i} g_{2}^{t} \mid \imath=1,2,3\right\}$. Then we have

$$
\begin{aligned}
& g_{1}^{-1} d g_{1}=\frac{1}{2}\left(\begin{array}{cc}
\omega^{1}-\boldsymbol{\omega}_{2}^{3} & \boldsymbol{\omega}_{2}^{1}+\boldsymbol{\omega}_{3}^{1}+\boldsymbol{\omega}^{2}-\boldsymbol{\omega}^{3} \\
-\boldsymbol{\omega}_{2}^{1}+\boldsymbol{\omega}_{3}^{1}+\boldsymbol{\omega}^{2}+\boldsymbol{\omega}^{3} & -\boldsymbol{\omega}^{1}+\boldsymbol{\omega}_{2}^{3}
\end{array}\right), \\
& g_{2}^{-1} d g_{2}=\frac{1}{2}\left(\begin{array}{cc}
\boldsymbol{\omega}^{1}+\boldsymbol{\omega}_{2}^{3} & \boldsymbol{\omega}_{2}^{1}-\boldsymbol{\omega}_{3}^{1}+\boldsymbol{\omega}^{2}+\boldsymbol{\omega}^{3} \\
-\boldsymbol{\omega}_{2}^{1}-\boldsymbol{\omega}_{3}^{1}+\boldsymbol{\omega}^{2}-\boldsymbol{\omega}^{3} & -\boldsymbol{\omega}^{1}-\boldsymbol{\omega}_{2}^{3}
\end{array}\right) .
\end{aligned}
$$

Proof. From $e_{\alpha}=g_{1} \underline{e}_{\alpha} g_{2}^{t}$, we get

$$
d e_{\alpha}=g_{1}\left[g_{1}^{-1} d g_{1} \underline{e}_{\alpha}+\underline{e}_{\alpha}\left(g_{2}^{-1} d g_{2}\right)^{t}\right] g_{2}^{t} .
$$

On the other hand, by (2.6) we have

$$
d e_{\alpha}=\sum \omega_{\alpha}^{\beta} e_{\beta}=\sum g_{1}\left(\omega_{\alpha}^{\beta} \underline{e}_{\beta}\right) g_{2}^{t} .
$$

From these two equations and noting that $\omega_{4}^{2}=\omega^{2}=\omega_{\imath}^{4} \varepsilon_{\imath}$, we can easily verify the lemma by direct computation.

\section{Timelike surface theory in $H_{1}^{3}$ and the case $H=1$}

Throughout this section, $M$ will denote an oriented connected smooth 2dimensional manifold, and $f: M \rightarrow H_{1}^{3}$ will be a timelike smooth immersion.

We let $\mathscr{F}_{f}^{(1)} \subset M \times \mathscr{F}$ denote the first order frame bundle of $f$. Thus $\left(m ; e_{1}\right.$, $\left.e_{2}, e_{3}, e_{4}\right) \in \mathscr{F}_{f}^{(1)}$ if $e_{4}=f(m)$ and $e_{2} \wedge e_{3}=f_{*}\left(T_{m} M\right)$ as oriented 2-plane. We restrict all forms and maps to $\mathscr{F}_{f}^{(1)}$. It follows that $e_{1} \in T_{f(m)} H_{1}^{3}$ is the oriented united normal to $f_{*}\left(T_{m} M\right)$ and hence, we may regard $e_{1}$ as well-defined as a map $e_{1}: M \rightarrow R_{2}^{4}$.

We have $\left\langle e_{1}, d f\right\rangle=\omega^{1}=0$, so the induced metric by $f$ on $M$ is $d s_{f}^{2}=\left(\omega^{2}\right)^{2}-$ $\left(\omega^{3}\right)^{2}$, and the structure equations for immersion $f$ are given as follows: 


$$
\begin{aligned}
& d \omega^{2}=-\omega_{3}^{2} \wedge \omega^{3}, \\
& d \omega^{3}=-\omega_{2}^{3} \wedge \omega^{2}, \\
& d \omega_{3}^{2}=\omega^{2} \wedge \omega^{3}+\omega_{2}^{1} \wedge \omega_{3}^{1} .
\end{aligned}
$$

Since $d \omega^{1}=\omega^{2} \wedge \omega_{2}^{1}+\omega^{3} \wedge \omega_{3}^{1}=0$, it follows that there exist smooth functions $h_{\imath j}=$ $h_{j i}(i, j=2,3)$ so that

$$
\begin{aligned}
& \omega_{2}^{1}=h_{22} \omega^{2}-h_{23} \omega^{3}, \\
& \omega_{3}^{1}=-h_{32} \omega^{2}+h_{33} \omega^{3} .
\end{aligned}
$$

One easily checks that $I I=h_{22}\left(\omega^{2}\right)^{2}-2 h_{23} \omega^{2} \omega^{3}+h_{33}\left(\omega^{3}\right)^{2}$ is a well-defined smooth quadratic form on $M$, which is called the second fundamental form. Its trace with respect to $d s_{f}^{2}$ denoted by $H=\left(h_{22}-h_{33}\right) / 2$ is defined as mean curvature of immersion $f$. It's easily checked that the function $H$ is a well-defined smooth function on $M$.

After the above preparation, we now set up to establish our main theorems.

THEOREM 3.1. Let $U \subseteq R^{1,1}$ be a domain in 2-dimentional Lorentz-Minkowskl space $R^{1,1}$ and $\{\eta, \xi\}$ be the global oriented null coordinates on $R^{1,1}$. Let $g_{1}, g_{2}$ : $U \rightarrow S L(2, R)$ be two maps satısfying the following three conditions:
(1) $\frac{\partial g_{1}}{\partial \xi}=\frac{\partial g_{2}}{\partial \eta}=0$,
(2) $\operatorname{det}\left(g_{1}^{-1} d g_{1}\right)=\operatorname{det}\left(g_{2}^{-1} d g_{2}\right)=0$,
(3) $\operatorname{det}\left(g_{1}^{-1} d g_{1}+\left(g_{2}^{-1} d g_{2}\right)^{t}\right) \neq 0$.

Then the map $f=g_{1} g_{2}^{t}: U \subseteq R^{1,1} \rightarrow H_{1}^{3}$ is a conformal (timelike) immersion with the mean curvature one.

Proof. Let $\left(e_{2}=g_{1} \underline{e}_{i} g_{2}^{t}\right)$ be the orthonormal frame associated to $g_{1}, g_{2}$. Under this frame, we have canonical 1 -forms $\left\{\omega^{2}, \omega_{j}^{2} \mid i, j=1,2,3\right\}$.

Denote by

By Lemma 2.1, we have

$$
\begin{aligned}
& \pi_{1}=\omega_{3}^{1}-\omega_{2}^{1}+\omega^{2}+\omega^{3}, \\
& \pi_{2}=\omega_{3}^{1}+\omega_{2}^{1}-\omega^{2}+\omega^{3}, \\
& \omega^{+}=\omega^{2}+\omega^{3}, \\
& \omega^{-}=\omega^{2}-\omega^{3} .
\end{aligned}
$$

$$
g_{1}^{-1} d g_{1}=\frac{1}{2}\left(\begin{array}{cc}
\omega^{1}-\omega_{2}^{3} & \pi_{2}+2 \omega^{-} \\
\pi_{1} & -\omega^{1}+\omega_{2}^{3}
\end{array}\right)
$$

and

$$
g_{2}^{-1} d g_{2}=\frac{1}{2}\left(\begin{array}{cc}
\omega^{1}+\omega_{2}^{3} & -\pi_{1}+2 \omega^{+} \\
-\pi_{2} & -\omega^{1}-\omega_{2}^{3}
\end{array}\right)
$$


By condition (1), we assume that

$$
\begin{aligned}
\omega_{1}^{4}-\omega_{2}^{3}=2 \alpha_{1}=2 A_{1}(\eta) d \eta, & \omega_{1}^{4}+\omega_{2}^{3}=2 \alpha_{2}=2 A_{2}(\xi) d \xi, \\
\pi_{1}=2 \gamma_{1}=2 C_{1}(\eta) d \eta, & -\pi_{2}=2 \gamma_{2}=2 C_{2}(\xi) d \xi, \\
\pi_{2}+2 \omega^{-}=2 \beta_{1}=2 B_{1}(\eta) d \eta, & -\pi_{1}+2 \omega^{+}=2 \beta_{2}=2 B_{2}(\xi) d \xi .
\end{aligned}
$$

Condition (2) means

$$
\alpha_{1}^{2}+\beta_{1} \gamma_{1}=\alpha_{2}^{2}+\beta_{2} \gamma_{2}=0 .
$$

So the induced metric $d s_{f}^{2}$ on $U$ is

$$
d s_{f}^{2}=\langle d f, d f\rangle=\left\langle d\left(g_{1} g_{2}^{t}\right), d\left(g_{1} g_{2}^{t}\right)\right\rangle=-\operatorname{det}\left(g_{1}^{-1} d g_{1}+\left(g_{2}^{-1} d g_{2}\right)^{t}\right) \neq 0 .
$$

More precisely,

$$
d s_{f}^{2}=2 \alpha_{1} \alpha_{2}+\beta_{1} \beta_{2}+\gamma_{1} \gamma_{2}=\left(2 A_{1} A_{2}+B_{1} B_{2}+C_{1} C_{2}\right) d \eta d \xi .
$$

It then follows that $f=g_{1} g_{2}^{t}: U \rightarrow H_{1}^{3}$ is a conformal (timelike) immersion.

We will now show that for this immersion $H=1$ by computing $H$ in a first order adapted frame. Without lose its generity, we assume that $2 A_{1} A_{2}+B_{1} B_{2}$ $+C_{1} C_{2}>0$, then we can write down that $d s_{f}^{2}=\lambda^{2} d \eta d \xi$ on $U$, for some smooth function $\lambda>0$ on $U$. From (3.3) and (3.4), we have

$$
A_{i}^{2}+B_{i} C_{\imath}=0 \text {, }
$$

for $i=1,2$ and

$$
2 A_{1} A_{2}+B_{1} B_{2}+C_{1} C_{2}=\lambda^{2} .
$$

First we will prove an assertion.

AsSERTION. For any $p \in U$, if there exist a neighborhood $V$ of $p$ in $U$ and some smooth functions $p_{i}, q_{\imath}(i=1,2)$ on $V$ so that

$$
\begin{aligned}
& A_{i}=\lambda p_{i} q_{\imath} \\
& B_{\imath}=\operatorname{sign}\left(B_{\imath}\right) \lambda p_{\imath}^{2} \\
& C_{\imath}=\operatorname{sign}\left(C_{\imath}\right) \lambda q_{\imath}^{2}
\end{aligned}
$$

for $i=1,2$, then $H(p)=1$.

Note that $B_{i} C_{\imath}=-A_{\imath}^{2} \leqq 0$, and hence $\operatorname{sign}\left(B_{\imath}\right) \operatorname{sign}\left(C_{\imath}\right)=-1$. For simplicity, we assume that $\operatorname{sign}\left(B_{\imath}\right)=1, \operatorname{sign}\left(C_{\imath}\right)=-1$ and $p_{1} p_{2}+q_{1} q_{2}=1$ from (3.6) and (3.7).

Let $h: V \rightarrow S L(2, R)$ be defined by

$$
h=\left(\begin{array}{cc}
p_{1} & q_{2} \\
-q_{1} & p_{2}
\end{array}\right),
$$

then $e_{4}\left(g_{1} h, g_{2}\left(h^{t}\right)^{-1}\right)=e_{4}\left(g_{1}, g_{2}\right)=g_{1} g_{2}^{t}$. Moreover we compute that 


$$
\begin{aligned}
\left(g_{1} h\right)^{-1} d\left(g_{1} h\right) & =h^{-1}\left(g_{1}^{-1} d g_{1}\right) h+h^{-1} d h \\
& =\left[\begin{array}{cc}
q_{2} d q_{1}+p_{2} d p_{1} & -q_{2} d p_{2}+p_{2} d q_{2}+\lambda d \eta \\
-p_{1} d q_{1}+q_{1} d p_{1} & p_{1} d p_{2}+q_{1} d q_{2}
\end{array}\right],
\end{aligned}
$$

and

$$
\begin{aligned}
\left(g_{2}\left(h^{-1}\right)^{t}\right)^{-1} d\left(g_{2}\left(h^{-1}\right)^{t}\right) & =h^{t}\left(g_{2}^{-1} d g_{2}\right)\left(h^{t}\right)^{-1}-\left(h^{-1} d h\right)^{t} \\
& =-\left[\begin{array}{cc}
q_{2} d q_{1}+p_{2} d p_{1} & -p_{1} d q_{1}+q_{1} d p_{1}-\lambda d \xi \\
-q_{2} d p_{2}+p_{2} d q_{2} & p_{1} d p_{2}+q_{1} d q_{2}
\end{array}\right] .
\end{aligned}
$$

Also denote the 1 -forms $\left\{\omega^{2}, \omega_{j}^{2} \mid i, j=1,2,3\right\}$ be the canonical 1-forms associated to the frame $\left\{e_{\alpha}\left(g_{1} h, g_{2}\left(h^{-1}\right)^{t}\right) \mid \alpha=1, \cdots, 4\right\}$. By Lemma 2.1, it follows that

and

$$
\omega^{1}=0, \quad \omega^{-}=\omega^{2}-\omega^{3}=\lambda d \eta, \quad \omega^{+}=\omega^{2}+\omega^{3}=\lambda d \xi
$$

$$
\pi_{1}=-\omega_{2}^{1}+\omega_{3}^{1}+\omega^{2}+\omega^{3}=2\left(-p_{1} d q_{1}+q_{1} d p_{1}\right) .
$$

Thus $\left\{e_{\alpha}\left(g_{1} h, g_{2}\left(h^{-1}\right)^{t}\right) \mid \alpha=1, \cdots, 4\right\}$ is an oriented adapted frame field on $V$ for immersion $f=g_{1} g_{2}^{t}$. The 1 -form $-p_{1} d q_{1}+q_{1} d p_{1}$ must have the form $\Phi d \eta$ for some function $\Phi$ on $V$, since we have the representations

$$
-p_{1} d q_{1}+q_{1} d p_{1}=\left\{\begin{array}{l}
-p_{1}^{2} d \frac{q_{1}}{p_{1}}=-p_{1}^{2} d\left(\frac{A_{1}(\eta)}{B_{1}(\eta)}\right) \quad \text { where } \quad p_{1} \neq 0, \\
q_{1}^{2} d \frac{p_{1}}{q_{1}}=-q_{1}^{2} d\left(\frac{A_{1}(\eta)}{C_{1}(\eta)}\right) \quad \text { where } q_{1} \neq 0 .
\end{array}\right.
$$

By the following Lemma 3.2, we conclude that assertion holds.

Now we continue to prove our theorem. Let

and

$$
U_{i}=\left\{p \in U \mid B_{i}(p) \neq 0\right\}
$$

for $i=1,2$.

$$
V_{\imath}=\left\{p \in U \mid C_{i}(p) \neq 0\right\}
$$

For $p \in U_{1} \cap U_{2} \cap V_{1} \cap V_{2}$, we can choose a neighborhood $V$ of $p$ such that $\left.B_{\imath}\right|_{V} \neq 0$ and $\left.C_{\imath}\right|_{V} \neq 0$, for $i=1,2$. Let $p_{i}=\operatorname{sign}\left(A_{\imath}\right) \sqrt{\left|B_{\imath}\right| / \lambda}$ and $q_{\imath}=\sqrt{\left|C_{\imath}\right| / \lambda}$ by assertion we conclude that $H=1$ holds on $U_{1} \cap U_{2} \cap V_{1} \cap V_{2}$.

Next by the continuity of the mean curvature function $H$, we have that $H=1$ holds on $\bar{U}_{1} \cap U_{2} \cap V_{1} \cap V_{2} \subseteq \overline{U_{1} \cap U_{2} \cap V_{1} \cap V_{2}}$. On the other hand, for $p \in$ $\left(U \backslash \bar{U}_{1}\right) \cap U_{2} \cap V_{1} \cap V_{2}$, we can choose a neighborhood $V$ of $p$ such that $\left.B_{1}\right|_{V}=0$, $\left.B_{2}\right|_{V} \neq 0$ and $\left.C_{\imath}\right|_{V} \neq 0$ for $i=1,2$. Let $p_{1}=0, \quad p_{2}=\operatorname{sign}\left(A_{2}\right) \sqrt{\left|B_{2}\right| / \lambda}$ and $q_{2}=$ $\sqrt{\left|C_{\imath}\right| / \lambda}$, by assertion we conclude that $H=1$ holds on $\left(U \backslash \bar{U}_{1}\right) \cap U_{2} \cap V_{1} \cap V_{2}$. So we see that $H=1$ holds on $U_{2} \cap V_{1} \cap V_{2}$.

Repeat the above discussion, we conclude that $H=1$ holds on $V_{1} \cap V_{2}$, on $V_{2}$ and finally on $U$.

LEMMA 3.2. Let $f: U \subseteq R^{1,1} \rightarrow H_{1}^{3}$ be an conformal (timelike) immession and 
$\{\eta, \xi\}$ be the global oriented null coordinates on $R^{1,1}$. Let $\left\{\omega^{2}, \omega_{j}^{2} \mid i, j=1,2,3\right\}$ be the canonical 1-forms associated with an oriented adapted frame field $\left\{e_{i} \mid i=\right.$ $1,2,3\}$. Then the immersion $f$ has mean curvature one if and only if $-\omega_{2}^{1}+\omega_{3}^{1}$ $+\omega^{2}+\omega^{3}$ has the form $\Phi d \eta$ for some function $\Phi$ on $U$.

Proof. Let $d s_{f}=\lambda^{2} d \eta d \xi$ denote the induced metric on $U$, so we have $\omega^{2}-\omega^{3}=\lambda d \eta$ and $\omega^{2}+\omega^{3}=\lambda d \xi$. We compute that

and

$$
\begin{aligned}
-\omega_{2}^{1}+\omega_{3}^{1} & =-\left(h_{22} \omega^{2}-h_{23} \omega^{3}\right)+\left(-h_{23} \omega^{2}+h_{33} \omega^{3}\right) \\
& =-2 H \omega^{3}-\left(h_{23}+h_{33}\right)\left(\omega^{2}-\omega^{3}\right),
\end{aligned}
$$

$$
\begin{aligned}
-\omega_{2}^{1}+\omega_{3}^{1}+\omega^{2}+\omega^{3} & =-2(H-1) \omega^{3}-\left(-1+h_{33}+h_{23}\right)\left(\omega^{2}-\omega^{3}\right) \\
& =-2(H-1) \omega^{3}-\lambda\left(-1+h_{33}+h_{23}\right) d \eta .
\end{aligned}
$$

So it then follows that lemma holds.

To complete the representation for the timelike surfaces with mean curvature one in $H_{1}^{3}$, we shall prove the following theorem.

THEOREM 3.3. Let $U \subseteq R^{1,1}$ be a simply connected domain and $f: U \rightarrow H_{1}^{3}$ be a conformal (timelike) immersion with mean curvature one. Then there exist two maps $F_{1}, F_{2}: U \rightarrow S L(2, R)$ satisfying condition (1), (2) and (3) such that

$$
f=F_{1} F_{2}^{t} .
$$

Proof. Let $d s_{f}^{2}=\lambda^{2} d \eta d \xi$ be the induced metric on $U$, and $e_{1}, e_{2}, e_{3}$ be the adapted frame fields on $U$ such that $e_{1}$ is the unit normal vecter field of $f$ in $H_{1}^{3}$. Then $\left\{e_{1}, e_{2}, e_{3}, e_{4}=f\right\}$ is a frame field of $R_{2}^{4}$. By the fact that $U$ is simply connected, we have the lifting maps $g_{1}, g_{2}: U \rightarrow S L(2, R)$ such that $e_{i}\left(g_{1}\right.$, $\left.g_{2}\right)=e_{2}$ for $i=1,2,3$ and $f=g_{1} g_{2}^{t}$. Again let $\left\{\omega^{2}, \omega_{j}^{2} \mid i, j=1,2,3\right\}$ be the canonical 1-forms associated to the frame field $\left\{e_{2} \mid i=1,2,3\right\}$. By Lemma 2.1 and $\omega^{1}=0$, we have

$$
\begin{aligned}
& g_{1}^{-1} d g_{1}=\frac{1}{2}\left(\begin{array}{cc}
-\omega_{2}^{3} & \omega_{2}^{1}+\omega_{3}^{1}+\omega^{2}-\omega^{3} \\
-\omega_{2}^{1}+\omega_{3}^{1}+\omega^{2}+\omega^{3} & \omega_{2}^{3}
\end{array}\right), \\
& g_{2}^{-1} d g_{2}=\frac{1}{2}\left(\begin{array}{cc}
\omega_{2}^{3} & \omega_{2}^{1}-\omega_{3}^{1}+\omega^{2}+\omega^{3} \\
-\omega_{2}^{1}-\omega_{3}^{1}+\omega^{2}-\omega^{3} & -\omega_{2}^{3}
\end{array}\right) .
\end{aligned}
$$

Consider the $\mathfrak{g l}(2, R)$-valued 1 -form $\mu$ on $U$ :

$$
\mu=\frac{1}{2}\left(\begin{array}{cc}
-\omega_{2}^{3} & \omega_{2}^{1}+\omega_{3}^{1}-\omega^{2}+\omega^{3} \\
-\omega_{2}^{1}+\omega_{3}^{1}+\omega^{2}+\omega^{3} & \omega_{2}^{3}
\end{array}\right) .
$$

It is easy to see that $\mu$ satisfies $d \mu=-\mu \wedge \mu$ (since $f$ has mean curvature one). It follows by the Frobenuis theorem that there exists a smooth map $h: U \rightarrow$ $S L(2, R)$ so that $\mu=h^{-1} d h$. 
Let us write

$$
h=\left(\begin{array}{ll}
a & b \\
c & d
\end{array}\right)
$$

for smooth functions $a, b, c$ and $d$ on $U$. Then if we set $F_{1}=g_{1} h^{-1}$ and $F_{2}=$ $g_{2} h^{t}$, by the fact that $\omega^{2}-\omega^{3}=\lambda d \eta$ and $\omega^{2}+\omega^{3}=\lambda d \xi$, we easily compute

$$
\begin{aligned}
& F_{1}^{-1} d F_{1}=\left(\begin{array}{cc}
-a c & a^{2} \\
-c^{2} & a c
\end{array}\right) \lambda d \eta, \\
& F_{2}^{-1} d F_{2}=\left(\begin{array}{cc}
b d & d^{2} \\
-b^{2} & -b d
\end{array}\right) \lambda d \xi .
\end{aligned}
$$

Since $d F_{1}\left(\right.$ resp. $\left.d F_{2}\right)$ has the form $\Phi d \eta($ resp. $\Psi d \xi)$ for $g l(2, R)$-valued function $\Phi$ (resp. $\Psi$ ), we must have that $F_{1}$ and $F_{2}$ satisfy the condition (1). Clearly, $F_{1}$ and $F_{2}$ also satisfy condition (2), (3) and

$$
F_{1} F_{2}^{t}=g_{1} g_{2}^{t}=f \text {. }
$$

This completes our proof.

\section{Representation formula and examples}

Let $U \subseteq R^{1,1}$ be a simply connected domain and $\{\eta, \xi\}$ be the global null coordinates on $R^{1,1}$. For given smooth functions $\alpha_{i}(\eta), \beta_{i}(\xi)(i=1,2,3)$ on $U$, satisfying

$$
\begin{aligned}
& \alpha_{1} \alpha_{2}+\alpha_{3}^{2}=0 \\
& \beta_{1} \beta_{2}+\beta_{3}^{2}=0,
\end{aligned}
$$

by Frobenuis theorem, there exist two maps

such that

$$
\begin{aligned}
& A(\eta): U \longrightarrow S L(2, R), \\
& B(\xi): U \longrightarrow S L(2, R),
\end{aligned}
$$

$$
\begin{aligned}
& A(\eta)^{-1} d A(\eta)=\left(\begin{array}{cc}
\alpha_{3} & \alpha_{1} \\
\alpha_{2} & -\alpha_{3}
\end{array}\right) d \eta \\
& B(\xi)^{-1} d B(\xi)=\left(\begin{array}{cc}
\beta_{3} & \beta_{1} \\
\beta_{2} & -\beta_{3}
\end{array}\right) d \xi .
\end{aligned}
$$

Then we obtain that a mapping given by

$$
f(\xi, \eta)=A(\eta) B(\xi)^{t}: U \longrightarrow H_{1}^{3}
$$

is a branched conformal (timelike) immersion with mean curvature one.

The mapping $f$ is an immersion if the functions $\alpha_{i}(\eta)$ and $\beta_{i}(\tilde{\xi})(i=1,2,3)$ 
satisfy

$$
\alpha_{1} \beta_{1}+\alpha_{2} \beta_{2}+2 \alpha_{3} \beta_{3} \neq 0
$$

At last we shall give here some examples.

Examples. 1) Let $\alpha_{1}=\beta_{1}=-1, \alpha_{2}=\alpha_{3}=\beta_{2}=\beta_{3}=1$. By solving (4.1) and (4.2), it follows that

$$
\begin{aligned}
& A(\eta)=\left(\begin{array}{cc}
\eta+1 & -\eta \\
\eta & -\eta+1
\end{array}\right), \\
& B(\xi)=\left(\begin{array}{cc}
\xi+1 & -\xi \\
\xi & -\xi+1
\end{array}\right) .
\end{aligned}
$$

Hence an entire timelike immersion $f: R^{1,1} \rightarrow H_{1}^{3}$ with mean curvature one is given by

$$
f(\xi, \eta)=(\xi+\eta, 2 \xi \eta, \eta-\xi, 2 \xi \eta+1) .
$$

2) Let $\alpha_{1}=\beta_{1}=-1, \alpha_{3}=\eta, \beta_{3}=\xi$ and $\alpha_{2}=\eta^{2}, \beta_{2}=\xi^{2}$. We have by (4.1) and

$$
\begin{aligned}
& A(\eta)=\left(\begin{array}{cc}
\sin \eta-\eta \cos \eta & \cos \eta \\
-\cos \eta-\eta \sin \eta & \sin \eta
\end{array}\right), \\
& B(\xi)=\left(\begin{array}{cc}
\sin \xi-\xi \cos \xi & \cos \xi \\
-\cos \xi-\xi \sin \xi & \sin \xi
\end{array}\right) .
\end{aligned}
$$

Then we have a branched immersion $f: R^{1,1} \rightarrow H_{1}^{3}$ with mean curvature one:

$$
\begin{aligned}
& f(u, v)= \frac{1}{2}\left(-u \sin u+\frac{u^{2}-v^{2}}{4} \cos u, u \cos u+\frac{u^{2}-v^{2}}{4} \sin u,\right. \\
&\left.2 \sin v-v \cos v+\frac{u^{2}-v^{2}}{4} \sin v, 2 \cos v+v \sin v+\frac{u^{2}-v^{2}}{4} \cos v\right)
\end{aligned}
$$

where $u=\eta+\xi, v=\eta-\xi$. And $f$ is an immersion on domain

$$
U=\left\{(u, v) \in R^{1,1}: u^{2}-v^{2}+4 \neq 0\right\} \text {. }
$$

3) Let $\alpha_{1}=\beta_{1}=-1, \beta_{2}=\beta_{3}=1$ and $\alpha_{2}=\eta^{2}, \alpha_{3}=\eta$. By (4.1) and (4.2), we get that

$$
\begin{gathered}
A(\eta)=\left(\begin{array}{cc}
\sin \eta-\eta \cos \eta & \cos \eta \\
-\cos \eta-\eta \sin \eta & \sin \eta
\end{array}\right), \\
B(\xi)=\left(\begin{array}{cc}
\xi+1 & -\xi \\
\xi & -\xi+1
\end{array}\right) .
\end{gathered}
$$

Then we have a branched immersion $f: R^{1,1} \rightarrow H_{1}^{3}$ with mean curvature one given by $f(\eta, \xi)=(1 / 2)\left(x_{1}, \cdots, x_{4}\right)$, where 
350

$$
\begin{aligned}
& x_{1}=2 \xi \sin \eta-\eta \cos \eta+\xi \eta(\sin \eta-\cos \eta), \\
& x_{2}=-2 \xi \cos \eta-\eta \sin \eta-\xi \eta(\sin \eta+\cos \eta), \\
& x_{3}=-2 \cos \eta-(2 \xi+\eta) \sin \eta+\xi \eta(\cos \eta-\sin \eta), \\
& x_{4}=2 \sin \eta-(2 \xi+\eta) \cos \eta-\xi \eta(\sin \eta+\cos \eta) .
\end{aligned}
$$

And $f$ is an immersion on domain $U=\left\{(\xi, \eta) \in R^{1,1} ; \eta \neq-1\right\}$.

Acknowledgement. The author wishes to express his gratitude to Professors C.H. Gu, H.S. Hu and Y.L. Xin for their helpful advices and encouragements.

\title{
REFERENCES
}

[1] R.L. BRyANT, Surfaces of mean curvature one in hyperbolic space, Asterisque, 154-155 (1987), 321-347.

[2] C.H. Gu, The extremal surfaces in the 3-dimensional Mikowski space, Acta Math. Sinica (N.S.), 1 (1985), 173-180.

[3] D. A. Hoffman AND R. Osserman, The geometry of the generalized Gauss map, Mem. Amer. Math. Soc., 236 (1980).

[4] K. Kenmotsu, Weierstrass formula for surfaces of prescribed mean curvature, Math. Ann., 245 (1979), 89-99.

[5] O. Kobayashi, Maximal surfaces in the 3 -dimensional space $L^{3}$, Tokyo J. Math. 6 (1983), 297-309.

[6] B. Palmer, Spacelike constant mean curvature surfaces in pseudo-Riemannian space forms, Ann. Global Anal. Geom., 8 (1990), 217-226.

[7] M. Spivak, A Comprehensive Introduction to Differential Geometry, Publish or Perish, Berkeley, 1979.

[8] J.A. Wolf, Spaces of Constant Curvature, 3rd ed, McGraw-Hill, 1974.

\author{
Accounting Department \\ SCHOOL OF MANEGEMENT \\ FUdAN UNIVERSITY \\ SHANGHAI, 200433, \\ P. R. CHINA
}

